\title{
基于虚场法的铝合金各向异性屈服及硬化属性 参数同步表征
}

\author{
付佳伟 ${ }^{1}$ 马 臻 ${ }^{1}$ 聂祥棼 $^{2}$ 谢稳伟 $^{1}$ 齐乐华 $^{1}$
}

(1. 西北工业大学机电学院 西安 710072;

2. 空军工程大学航空等离子体动力学国家级实验室 西安 710038)

\begin{abstract}
摘要: 变形铝合金板材因轻质、高比强和比模量等优点广泛应用于航空航天工业中, 其轧制生产过程引起的塑性各向异性可 显著影响板材的变形行为, 加大零部件成形精度控制和服役行为数值模拟预测的难度。针对目前常规测试方法表征材料各向 异性屈服及各向异性塑性硬化属性所需试验数量多、种类复杂、限制条件多的现状, 结合全场变形测量和虚场法, 通过一种 桥型试件的循环拉伸-压缩试验, 首次实现 2024 铝合金板材各向异性屈服与塑性硬化本构参数的同步表征, 大幅减少试验数 量, 简化试验过程。研究表明, 采用当前的加载构型, 在参数优化目标函数中结合材料 $0^{\circ}$ 和 $90^{\circ}$ 两个拉伸加载方向的试验 数据, 并配合多虚场约束, 可以在不同参数表征初始猜测值下产生稳定的 Hill1948 各向异性屈服参数表征结果, 保证解的准 确性; 对于非线性运动硬化模型, 采用单材料方向加载和单虚场的目标函数即可获得对应材料方向稳定可靠的非线性运动硬 化参数表征结果。研究成果可为铝合金板材成形工艺分析提供理论依据、数据参考和便捷的测试技术支持。
\end{abstract}

关键词: 2024 铝合金; 塑性各向异性; 本构参数表征; 虚场法; 循环拉伸-压缩

中图分类号: TG339

\section{Identification of the Anisotropic Yield and Hardening Constitutive Parameters for Aluminum Alloys Using the Virtual Fields Method}

\author{
FU Jiawei $^{1}$ MA Zhen ${ }^{1}$ NIE Xiangfan ${ }^{2}$ XIE Wenwei ${ }^{1}$ QI Lehua ${ }^{1}$ \\ (1. School of Mechanical Engineering, Northwestern Polytechnical University, Xi'an 710072; \\ 2. Science and Technology on Plasma Dynamics Lab, Air Force Engineering University, Xi'an 710038)
}

\begin{abstract}
Wrought aluminum alloy sheets are widely applied in aeronautic and astronautic industries thanks to their lightweight, high specific strength and high specific rigidity. They exhibit notable anisotropic plasticity due to the rolling fabrication process, which can significantly influence the mechanical behavior of the sheets, but also bring complexity to the analytical and numerical study of various forming processes. Currently, the conventional identification methods for the anisotropic yield and hardening properties usually involve multiple homogeneous tests and complex testing machines. They are also constrained by various limitations. In order to simplify the identification procedures, the full-field deformation measurement and the virtual fields method are coupled to identify the anisotropic yield and hardening constitutive parameters simultaneously from the same cyclic tension-compression tests on the bridge-like 2024 aluminum alloy sheet specimens. The results show that using the current test configuration the Hill1948 anisotropic yield parameters can be identified successfully with stable results when the two tension stages in the rolling and the transverse directions are combined and the multiple virtual fields constraints applied. As for the nonlinear kinematic hardening parameters, loading in a single material direction and a single set of virtual fields can guarantee a reliable identification result. The current work provides useful material information and a convenient identification tool for the aluminum sheet metal forming industry.
\end{abstract}

* 国家自然科学基金(51805439)、航空等离子体动力学国家级实验室开放基 金(6142202190202)、陕西省高层次人才引进计划青年(00121)和中央高校 基本科研业务费(31020190503006)资助项目。20210203 收到初稿, 20210726 收到修改稿 
Key words: 2024 aluminum alloy; anisotropic plasticity; constitutive parameters identification; virtual fields method; cyclic tension-compression

\section{0 前言}

变形铝合金因其轻质、高比强度、比模量和良 好的延展性, 被广泛应用于航空、航天、汽车等领 域, 如飞机、航天器中大型整体复杂薄壁壳体、汽 车防撞梁总成等 ${ }^{[1-2]}$, 是实现装备减重、节能降耗的 优良材料。对于铝合金板材，在轧制时材料沿不同 方向的塑性流动相异, 形成有取向性的织构, 从而 引起板材塑性属性的各向异性 ${ }^{[3]}$ 。这种各向异性显 著影响板材的塑性变形行为和零件的成形结果, 如 成形件在不同方向上表现出不同程度的回弹、起皱 或制耳等现象 ${ }^{[4]}$ 。采用有限元数值模拟是金属成形 工业中优化工艺过程进而提高成形精度的有效手 段, 而适当的本构模型和准确的模型本构参数则是 成功开展有限元数值模拟的重要前提。

众所周知, 影响金属板壳件成形精度的板材回 弹、起皱等现象与材料在不同方向上的塑性屈服与 硬化规律密切关联。为了准确表征金属塑性各向异 性, 国内外学者建立了诸多本构模型。基于二次屈 服函数的经典 Hill1948 模型 ${ }^{[5]}$ 和 BARLAT 等 ${ }^{[6]}$ 建立 的高次屈服函数 YLD2000-2D 模型等可有效表征金 属的各向异性塑性屈服行为, 但这些模型仅适用于 单向加载条件。当载荷路径变化时, 金属可表现出 复杂的各向异性塑性硬化行为, 如反向加载时的提 前屈服(包辛格效应)和硬化强度水平下降(永久软化 效应)等, 此时, 上述模型将不再适用。针对此, FREDERICK 等 ${ }^{[7-9]}$ 通过建立可移动屈服面的运动硬 化模型, 实现对此类材料行为的有效表征。BARLAT 等 ${ }^{[10]}$ 则基于扭曲塑性理论建立了 $\mathrm{HAH}$ 模型, 通过 屈服面的有序扭曲和转动, 亦实现对材料各向异性 塑性硬化行为的准确预测。此外, 王海波等 ${ }^{[11]}$ 对 YLD2000-2D 和 A-F 运动硬化模型进行了改进, 实 现对非比例加载状态下铝合金板料变形行为的精确 描述。

上述各向异性模型通常包含数量众多的本构参 数。采用目前常规测试方法确定各向异性屈服参数 时, 需沿板材不同方向(如与板材轧制方向呈 $0^{\circ}$ 、

$45^{\circ}$ 和 $90^{\circ}$ 夹角方向)分别开展单向拉伸或压缩试 验。因部分参数之间存在耦合关系(如 YLD2000-2D 模型), 还需开展双轴拉伸或液压膨胀试验 ${ }^{[12-13]}$ 。常 规试验中试件处于单调的均匀应力应变状态, 从单
次试验中仅能提取单个方向的材料参数, 所选模 型各向异性参数的增加则意味着所需试验数量的 增加。对于各向异性塑性硬化, 通常采用循环拉 伸-压缩试验 ${ }^{[14-15]}$ 或循环剪切-反向剪切试验 ${ }^{[16]}$ 。为 减小压缩时试件的面外屈曲, 需配以专用的防屈曲 变形装置。由此可见, 为了全面表征材料的各向异 性参数, 不但需要开展数量众多的试验, 同时还需 使用不同试验机和试验装置, 过程复杂繁琐, 亦增 加了试验成本。不仅如此, 受试验条件限制, 常规 测试方法所基于的均匀状态假设往往难以完全满 足。例如, 压缩试验中的防屈曲装置和剪切试验中 的试件夹具等, 均可对试件产生局部效应, 破坏其 均匀应力应变状态, 给基于均匀状态假设的测试结 果引入误差。

随着数字图像相关 ${ }^{[17]}$ 等非接触式全场变形测 量技术的快速发展, 使用更为丰富的全场数据来研 究材料的力学行为已成为可能。ZHANG 等 ${ }^{[18]}$ 基于 有限元模型修正法从十字型试件双轴拉伸的变形场 数据中一次提取出 5000 系铝合金的 Bron-Besson 各 向异性屈服参数。作为一种行之有效的反问题求解 思路, 有限元模型修正法已得到广泛应用。但是, 这类方法在求解材料参数时需要循环修正有限元模 型以减小模型和试验测量之间的残差, 此过程运算量 大, 耗时长, 如在一个反向循环的简单条件下表征 HAH 模型参数时, 所需收敛时间可达数天之久 ${ }^{[16]}$ 。 由英国南安普顿大学 PIERRON 等 ${ }^{[19]}$ 提出的虚场法 则克服了上述缺点。该方法基于虚功原理, 充分利 用试件的全场变形测量数据, 通过试件弹塑性变形 过程中内、外虚功残差的最小化, 实现材料参数的 快速表征。利用虚场法, 可将 HAH 模型参数的表 征时间从数天减少至半小时以内 ${ }^{[20]}$ 。MARTINS 等 ${ }^{[21]}$ 则设计了特殊构型的十字型双轴拉伸模拟试验, 实 现了各向异性屈服模型的多参数一次表征。

综上所述, 为了准确预测铝合金板材在复杂成 形应力状态下的力学行为, 提高成形精度, 需要准 确知晓材料的各向异性屈服与塑性硬化本构参数。 采用反问题求解法相较于均匀状态的常规测试方法 能够大幅简化试验过程。本文采用一种桥型试件循 环拉伸-压缩加载构型, 结合全场变形测量和虚场 法, 首次以 2024 变形铝合金板材为对象, 仅靠单轴 万能试验机, 从同一力学试验中实现材料各向异性 屈服与塑性硬化属性的多参数同步表征, 最大程度 
地减少试验数量, 降低试验设备要求, 同时也避免 了使用不同试验机、不同试件所引入的各类系统误 差, 为铝合金板材成形工艺研究提供理论依据、数 据参考和便捷的测试技术支持。

\section{1 桥型试件循环拉压加载试验}

\section{1 试验材料}

选用厚度为 $2.5 \mathrm{~mm}$ 的 2024 铝合金板材为试验 材料, 其化学成分如表 1 所示。

表 12024 铝合金化学成分

$\%$

\begin{tabular}{cccccc}
\hline 化学成分 & $\mathrm{Cu}$ & $\mathrm{Si}$ & $\mathrm{Fe}$ & $\mathrm{Mn}$ & $\mathrm{Mg}$ \\
\hline 质量分数 & $3.8 \sim 4.9$ & 0.50 & 0.50 & $0.3 \sim 0.9$ & $1.2 \sim 1.8$ \\
\hline \hline 化学成分 & $\mathrm{Zn}$ & $\mathrm{Cr}$ & $\mathrm{Ti}$ & $\mathrm{Al}$ & \\
\hline 质量分数 & 0.25 & 0.10 & 0.15 & 其余 & \\
\hline
\end{tabular}

\section{2 试验方法}

为简化试验过程, 实现通过最少数量的力学试 验同步表征 2024 铝合金板材各向异性屈服与塑性 硬化本构参数, 需采用合适的加载构型。对于常用 的各向异性屈服模型, 其各向异性屈服参数与试件 的应力应变状态密切相关。例如, Hill1948 模型中, 部分参数与两个法向应力或法向应力的耦合作用相 关, 而其他参数则与剪切应力相关。在表征某一参 数时, 需使试件对应应力分量获得充足的应力水平。 因此, 若要从单次试验中同步表征材料的各向异性 屈服多参数, 被测试件应处于拉剪均衡的丰富应力
状态。另一方面，对于材料各向异性塑性硬化属性 表征，在开展薄板类试件拉伸-压缩试验时，当压缩 应力超过某一临界值时, 试件将失稳而发生屈曲, 打破平面应力状态，此时，基于平面应力假设的参 数表征将不再适用。因此, 要在更高的压缩塑性变 形水平下表征材料的各向异性塑性硬化属性，所采 用的试件加载构型还应尽可能不易发生屈曲。

基于以上两点, 本文采用了一种桥型试件的拉 伸-压缩循环加载模式。如图 1a 所示，该试件的变 形部分实质上可看作两个圆弧过渡的(避免应力集 中)剪切构型的对称组合。作者前期的数值研究表 明，相较于常见的等截面或双缺口试件，该桥型构 型可在单向拉-压载荷下产生显著水平的剪切应

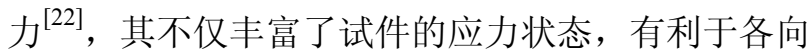
异性屈服多参数的一次表征，同时也减小了压缩正 应力, 从而有效延迟由压缩正应力引起的试件屈曲 的发生。

在 2024 铝合金板材上沿材料轧制成形方向 (图 1b)和垂直于轧制成形方向(图 1c)分别加工出桥 型试件(图 1d)。如图 2 所示, 使用万能试验机对 $0^{\circ}$ 和 $90^{\circ}$ 方向的试件分别开展两个拉伸-压缩循环的 加载试验。装夹试件时，应使试件对称中心线与夹 头中心线重合，从而保证试件变形过程中的对中性。 加载过程中, 采用 VIC-3D 数字图像相关系统获取 试件的全场变形数据。试验机横梁移动速率设定为 $0.01 \mathrm{~mm} / \mathrm{s}$, 对应名义应变率约为 $3 \times 10^{-4} \mathrm{~s}^{-1}$ 。为在 较高塑性应变水平下表征材料的各向异性屈服参数,

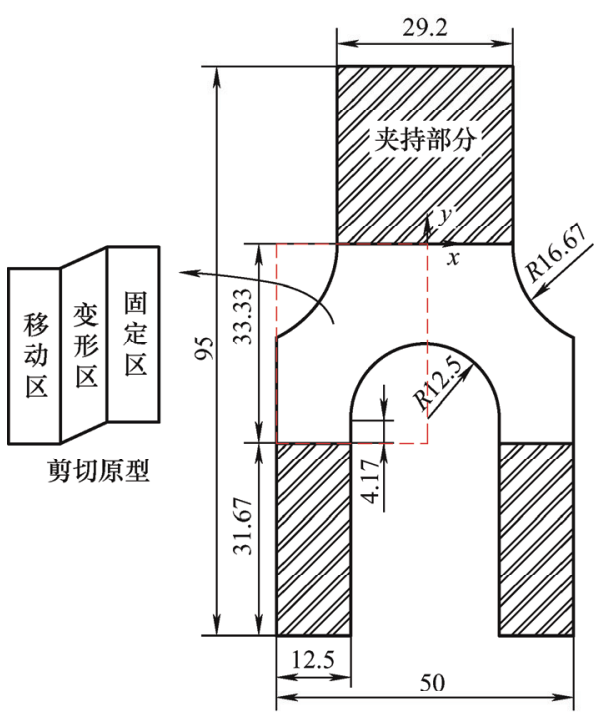

(a) 试件构型

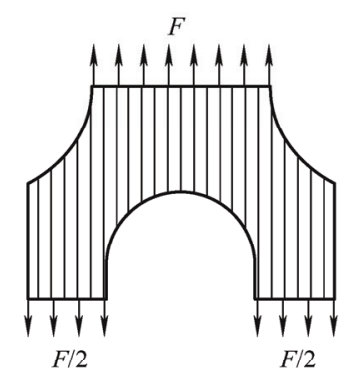

(b) $0^{\circ}$ 方向

$F$

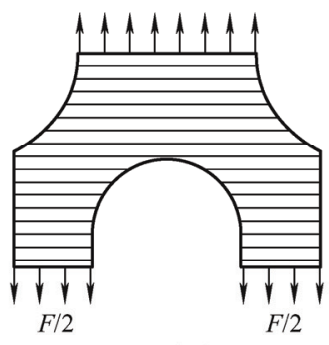

(c) $90^{\circ}$ 方向

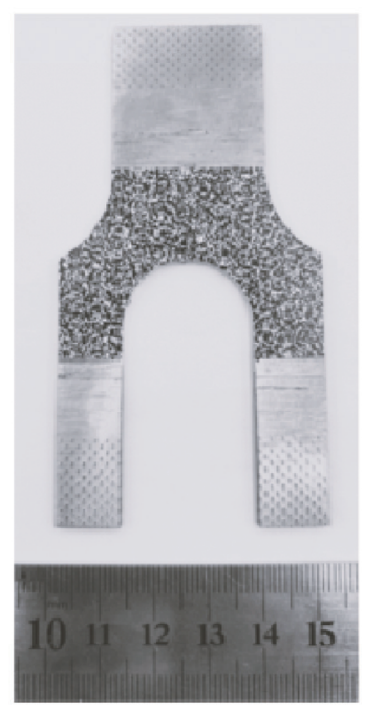

(d) 桥型试件

图 1 桥型试件及其加载构型 
试件第一个拉伸阶段的变形水平设定为高于后三个 阶段, 试件的压缩变形量设定为屈曲前的最高压缩 水平。图 3 所示为材料 $0^{\circ}$ 方向循环拉伸-压缩试验 力-位移曲线, 其中 $\mathrm{A} 1 、 \mathrm{~B} 1 、 \mathrm{~A} 2 、 \mathrm{~B} 2$ 分别代表第 一个拉伸、压缩和第二个拉伸、压缩阶段。为展示 屈曲的影响, B2 阶段的压缩变形量持续增加至发生 显著屈曲为止(B2 曲线上尧部分)。从曲线中可以 明显观察到由包辛格效应引起的材料提前屈服 (如 B1 阶段 $Y_{2}<Y_{1}$ )。试验时, 以 $1 \mathrm{~Hz}$ 为频率采集 变形试件的数字图像, 通过数字图像相关运算得 到试件的全场变形并计算出对应的对数应变场。 本文所采用的数字图像相关运算参数为: 子集大 小 $40 \times 40$ 像素, 子集间步长量 11 像素。基于静 止试件的数字图像相关运算, 可计算出该参数下 的应变测量分辨率约为 $1 \times 10^{-5}$ 。图 4 所示为 $\mathrm{A} 1$ 阶段终了时试件的位移场云图。

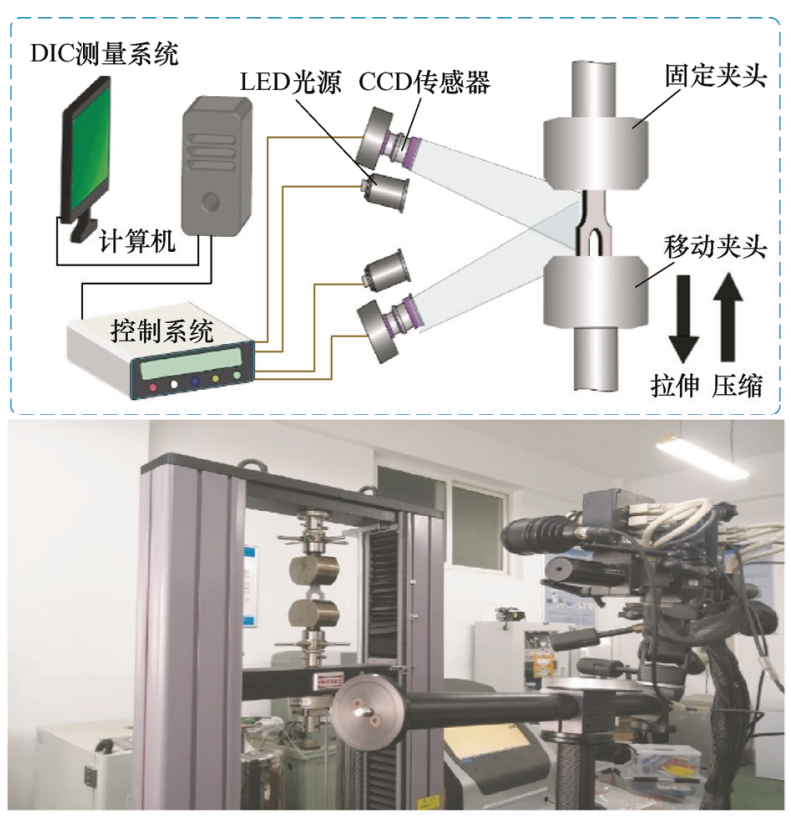

图 2 桥型试件循环拉伸-压缩试验测试系统及装置

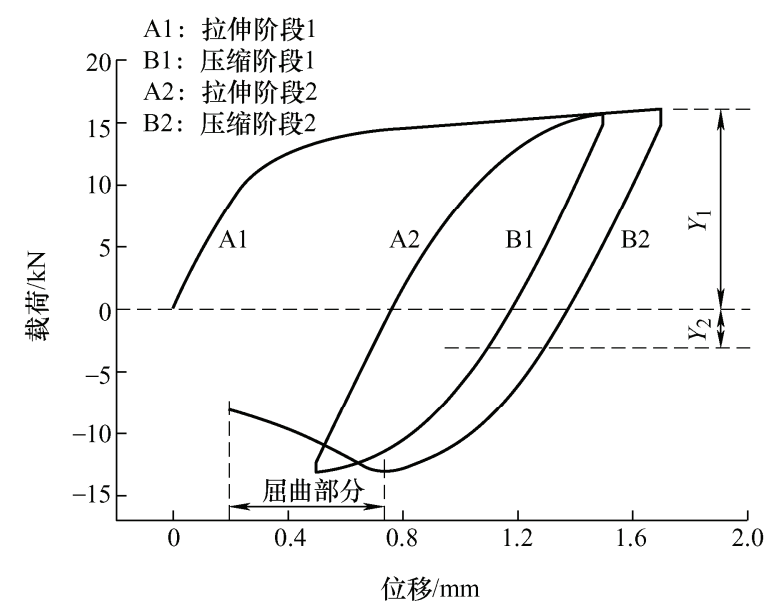

图 3 材料 $0^{\circ}$ 方向循环拉伸-压缩试验力-位移曲线

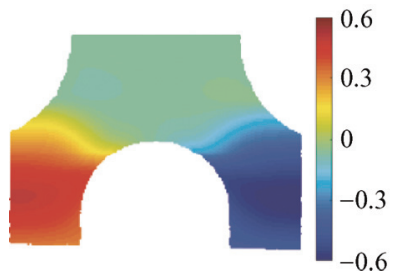

(a) $x$ 方向位移

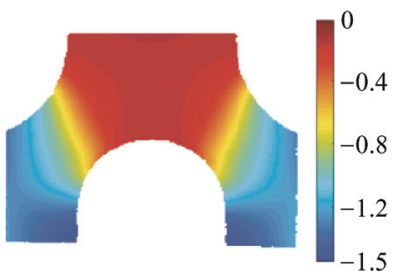

(b) $y$ 方向位移
图 4 第一拉伸阶段终了时试件的位移场云图 $(\mathrm{mm})$

\section{2 本构模型及参数表征方法}

\section{1 本构模型}

针对 2024 铝合金板材, 本文选用 Hill1948 屈服 准则和非线性运动硬化模型表征材料的各向异性屈 服与塑性硬化行为。对于平面应力状态, 材料的屈 服函数表达式为

$$
F\left(\sigma_{i j}, \varepsilon_{\mathrm{p}}\right)=\sigma_{\text {eq }}\left(\sigma_{i j}\right)-\sigma_{\mathrm{s}}\left(\varepsilon_{\mathrm{p}}\right)=0
$$

式中, $\sigma_{\text {eq }}$ 为等效应力, $\sigma_{\mathrm{s}}$ 为当前流动应力。对于 Hill1948 屈服准则 ${ }^{[5]}$

$$
\sigma_{\mathrm{eq}}=\sqrt{(H+G) \sigma_{x x}^{2}+(H+F) \sigma_{y y}^{2}-2 H \sigma_{x x} \sigma_{y y}+2 N \sigma_{x y}^{2}}
$$

式中, $H 、 G 、 F 、 N$ 为材料的各向异性屈服参数。 当前流动应力 $\sigma_{\mathrm{s}}$ 为等效塑性应变 $\varepsilon_{\mathrm{p}}$ 的函数, 对于常 规的单向加载, 采用 Swift 塑性硬化规律, 即

$$
\sigma_{\mathrm{s}}\left(\varepsilon_{\mathrm{p}}\right)=K\left(\varepsilon_{0}+\varepsilon_{\mathrm{p}}\right)^{n}
$$

式中, $K 、 \varepsilon_{0} 、 n$ 为塑性硬化参数。由于 $H+G=1$, 采用该屈服准则时, 需要表征的独立本构参数共 6 个，即 $H 、 F 、 N 、 K 、 \varepsilon_{0} 、 n$ 。

本文针对反向循环加载模式下铝合金各向异性 塑性硬化行为所选取的非线性运动硬化模型可表 示为

$$
F\left(\sigma, \alpha, \varepsilon_{\mathrm{p}}\right)=\sigma_{\text {eq }}(\boldsymbol{\sigma}-\boldsymbol{\alpha})-\sigma_{\mathrm{s}}\left(\varepsilon_{\mathrm{p}}\right)=0
$$

该模型中等效应力的计算基于 von Mises 屈服 准则, 其是应力张量 $\boldsymbol{\sigma}$ 与背应力张量 $\boldsymbol{\alpha}$ 的函数, 表 达式为

$$
\sigma_{\mathrm{eq}}(\sigma-\alpha)=\sqrt{\frac{3}{2}\left(s-\alpha^{\mathrm{dev}}\right):\left(s-\alpha^{\mathrm{dev}}\right)}
$$

式中, $\boldsymbol{s}$ 与 $\boldsymbol{\alpha}^{\mathrm{dev}}$ 分别表示偏应力张量和偏背应力张 量, “:”为张量内积符号。在背应力张量 $\boldsymbol{\alpha}$ 的作用 下，屈服面可在应力空间中实现移动，同时依照流 动应力 $\sigma_{s}$ 的变化规律均匀扩大。由 FREDERICK 等 ${ }^{[7]}$ 率先建立的非线性运动硬化模型, 其背应力张 
量的变化增量形式为

$$
\mathrm{d} \boldsymbol{\alpha}=\frac{C}{\sigma_{\mathrm{s}}}(\boldsymbol{\sigma}-\boldsymbol{\alpha}) \mathrm{d} \varepsilon_{\mathrm{p}}-\gamma \boldsymbol{\alpha} \mathrm{d} \varepsilon_{\mathrm{p}}
$$

式中, $C$ 与 $\gamma$ 分别为运动硬化模量和运动硬化下降 参数。CHABOCHE ${ }^{[8]}$ 通过将相同运动硬化形式进行 叠加, 提高了模型的适用性, 该模型表达式为

$$
\mathrm{d} \boldsymbol{\alpha}_{i}=\frac{C_{i}}{\sigma_{\mathrm{s}}}(\sigma-\alpha) \mathrm{d} \varepsilon_{\mathrm{p}}-\gamma_{i} \boldsymbol{\alpha}_{i} \mathrm{~d} \varepsilon_{\mathrm{p}}
$$

且

$$
\boldsymbol{\alpha}=\sum_{i=1}^{M} \boldsymbol{\alpha}_{i}
$$

同时, 屈服面的扩大采用 Voce 硬化规律, 即

$$
\sigma_{\mathrm{s}}\left(\varepsilon_{\mathrm{p}}\right)=Y_{0}+R_{\text {sat }}\left(1-\exp \left(-m \varepsilon_{\mathrm{p}}\right)\right)
$$

因此, 对于非线性运动硬化模型, 需要表征的 各向异性塑性硬化本构参数为 $C_{i} 、 \gamma_{i} 、 Y_{0} 、 R_{\mathrm{sat}}$ 和 $m$ 。

\section{2 应力计算}

明确应力-应变的本构关系是开展本构参数表 征的重要前提。在计算弹塑性变形过程中的应力时, 需要基于本构关系并通过应变值来确定应力值。本 文采用 SUTTON 等建立的应力推导算法 ${ }^{[23]}$, 根据 Hooke 定律有

$$
\mathrm{d} \boldsymbol{\sigma}=\boldsymbol{Q}: \mathrm{d} \boldsymbol{\varepsilon}^{\mathrm{e}}
$$

式中, $Q$ 为刚度矩阵, $\mathrm{d} \boldsymbol{\varepsilon}^{\mathrm{e}}$ 为弹性应变增量, $\mathrm{d} \boldsymbol{\varepsilon}^{\mathrm{e}}$ 可 由总应变增量 $\mathrm{d} \boldsymbol{\varepsilon}$ 和塑性应变增量 $\mathrm{d} \boldsymbol{\varepsilon}^{\mathrm{p}}$ 所替代, 即

$$
\mathrm{d} \boldsymbol{\sigma}=\boldsymbol{Q}:\left(\mathrm{d} \boldsymbol{\varepsilon}-\mathrm{d} \boldsymbol{\varepsilon}^{\mathrm{p}}\right)
$$

根据关联流动法则有

$$
\mathrm{d} \boldsymbol{\varepsilon}^{\mathrm{p}}=\mathrm{d} \lambda \frac{\partial F}{\partial \boldsymbol{\sigma}}
$$

式中, $\mathrm{d} \lambda$ 为塑性乘子。由一致性条件可知, 应力保 持在屈服面上, 即

$$
\mathrm{d} F=\frac{\partial F}{\partial \boldsymbol{\sigma}}: \mathrm{d} \boldsymbol{\sigma}+\frac{\partial F}{\partial \varepsilon_{\mathrm{p}}} \mathrm{d} \varepsilon_{\mathrm{p}}=0
$$

且

$$
\mathrm{d} \varepsilon_{\mathrm{p}}=\sqrt{\frac{2}{3} \mathrm{~d} \varepsilon^{\mathrm{p}}: \mathrm{d} \varepsilon^{\mathrm{p}}}
$$

将式(11)、(12)代入式(13), 有

$$
\mathrm{d} \lambda=\frac{\frac{\partial F}{\partial \boldsymbol{\sigma}}: \boldsymbol{Q}: \mathrm{d} \boldsymbol{\varepsilon}}{\frac{\partial F}{\partial \boldsymbol{\sigma}}: \boldsymbol{Q}: \frac{\partial F}{\partial \boldsymbol{\sigma}}-\frac{\partial F}{\partial \varepsilon_{\mathrm{p}}} \sqrt{\frac{2}{3} \frac{\partial F}{\partial \boldsymbol{\sigma}}: \frac{\partial F}{\partial \boldsymbol{\sigma}}}}
$$

结合式(11)、(12)和(15), 可推导出

$$
\mathrm{d} \boldsymbol{\sigma}=\left(\boldsymbol{Q}-\frac{\boldsymbol{Q}: \frac{\partial F}{\partial \boldsymbol{\sigma}} \otimes \boldsymbol{Q}: \frac{\partial F}{\partial \boldsymbol{\sigma}}}{\frac{\partial F}{\partial \boldsymbol{\sigma}}: \boldsymbol{Q}: \frac{\partial F}{\partial \boldsymbol{\sigma}}-\frac{\partial F}{\partial \varepsilon_{\mathrm{p}}} \sqrt{\frac{2}{3} \frac{\partial F}{\partial \boldsymbol{\sigma}}: \frac{\partial F}{\partial \boldsymbol{\sigma}}}}\right): \mathrm{d} \boldsymbol{\varepsilon}
$$

至此, 根据式(16), 应力的变化可由实际测量得到 的应变所推算出。

\section{3 虚场反问题求解法}

本文采用基于虚功原理的虚场法表征材料的各 向异性本构参数。对于任意一个体积为 $V$ 的连续可 变形固体，其在边界 $\partial V$ 上受到载荷向量 $\boldsymbol{T}$ 的作用, 当物体重力可忽略且载荷为准静态时, 有等式

$$
\underbrace{-\int_{V} \boldsymbol{\sigma}: \boldsymbol{\varepsilon}^{*} \mathrm{~d} V}_{\mathrm{IVW}}+\underbrace{\int_{\partial V} \boldsymbol{T} \cdot \boldsymbol{u}^{*} \mathrm{~d} S}_{\mathrm{EVW}}=0
$$

式中, $\boldsymbol{u}^{*}$ 为自行定义的虚位移向量, $\boldsymbol{\varepsilon}^{*}$ 为 $\boldsymbol{u}^{*}$ 求导 得到的虚应变张量。虚功原理表明, 物体各点内虚 功(等于各点内应力和虚应变的张量内积)求积分所 得到的总内虚功与总外虚功(等于各边界所受外载 荷和虚位移的向量内积再求积分)守恒。一个虚位移 场实质上是一个使得式(17)成立的检验函数, 它可 以是任意连续可微函数。实际中, 常将试件固定边 界上的虚位移限制为零, 以消除难以测量的固定边 界作用力对虚功的影响, 同时将移动边界上与载荷 方向一致的虚位移设置为常数, 从而将测量的载荷 纳入虚功计算之中。

对于弹-塑性变形, 可建立一个衡量变形全过程 内、外虚功总残差的目标函数

$$
C(\boldsymbol{X})=\sum_{j=1}^{P}\left(-\int_{V} \sigma(\boldsymbol{X}, \boldsymbol{\varepsilon}): \boldsymbol{\varepsilon}^{*} \mathrm{~d} V+\int_{\partial V} \boldsymbol{T} \cdot \boldsymbol{u}^{*} \mathrm{~d} S\right)_{j}^{2}
$$

式中, $j$ 表示第 $j$ 个加载步, 共 $P$ 个加载步。对于薄 板试件，可看作平面应力状态，因而有

$$
C(\boldsymbol{X})=\sum_{j=1}^{P}\left(-t \int_{A} \sigma(\boldsymbol{X}, \boldsymbol{\varepsilon}): \boldsymbol{\varepsilon}^{*} \mathrm{~d} S+\int_{\partial V} \boldsymbol{T} \cdot \boldsymbol{u}^{*} \mathrm{~d} S\right)_{j}^{2}
$$

式中, $t$ 为试件厚度, $A$ 为试件测量区域面积。由于 目标函数中, 除向量 $\boldsymbol{X}$ 所表示的本构参数为未知, 其余各部分均为已知(通过测量或自定义得到), 因 此, 给定一组本构参数的初始猜测值, 通过循环迭 代修正来最小化目标函数即可实现本构参数的快速 表征。本研究使用 Matlab ${ }^{\circledR}$ 编写计算程序, 采用非线 性最小二乘法实现目标函数最优化。

图 5 所示为 2024 铝合金本构参数表征流程图。 首先, 按照第 1.2 节所述分别沿材料 $0^{\circ}$ 和 $90^{\circ}$ 方 向对桥型试件开展循环拉伸-压缩试验, 采集全场变 
形和载荷数据。随后, 将应变场和载荷数据代入参 数表征程序, 用于计算试件每个加载步的内虚功、

外虚功及其之间的残差。输入待求参数的初始猜测 值以启动参数优化过程, 待目标函数收玫后获得参 数表征结果。对于各向异性屈服参数, 仅需使用第 一个拉伸阶段的试验数据。由于结合多加载方向和 多虚场约束可显著提高各向异性屈服参数表征结果 的收玫性和准确性 ${ }^{[24]}$, 本研究在表征 Hill1948 模型 参数时, 结合了 $0^{\circ}$ 和 $90^{\circ}$ 两次试验第一个拉伸阶 段的试验数据。定义虚场时, 为便于虚功计算, 所 选虚场满足试件上端边界虚位移为零且下端边界虚 位移为等值的边界条件。采用幂函数、三角函数等 基本函数形式的组合定义了多组独立虚场, 以提高
各向异性屈服参数表征结果的稳定性 ${ }^{[19]}$ 。所采用的 多虚场形式为

$$
\left\{\begin{array}{l}
\mathrm{VF} 1: u_{x}^{*}=0, u_{y}^{*}=y \\
\mathrm{VF} 2: u_{x}^{*}=\sin \left(\frac{\pi x}{\min (y)}\right) \cos \left(\frac{\pi y}{\min (y)}\right), u_{y}^{*}=y \\
\mathrm{VF} 3: u_{x}^{*}=\frac{\sin (x y(y-\min (y)))}{100}, u_{y}^{*}=y
\end{array}\right.
$$

对于运动硬化模型, 使用完整的两个拉伸-压缩循 环进行参数表征。前期数值研究表明, 单方向加 载、单虚场条件即可实现运动硬化模型参数的准 确表征 $^{[22]}$ 。

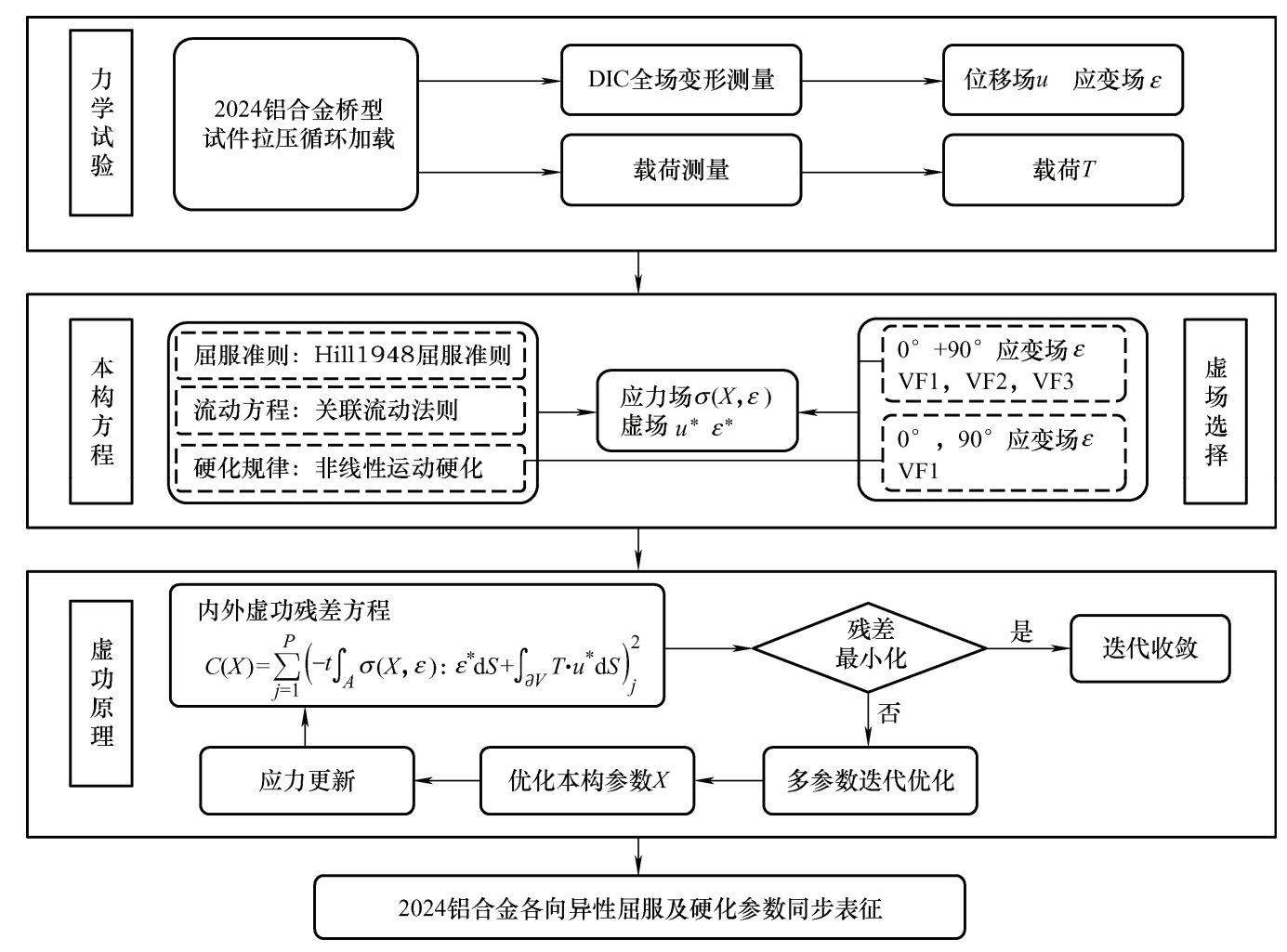

图 52024 铝合金各向异性塑性本构参数表征流程图

\section{3 结果与讨论}

\subsection{Hill1948 模型参数表征结果}

表 2 所示为目标函数包含双加载方向 $\left(0^{\circ}\right.$ 和 $90^{\circ}$ 方向)和三组虚场(VF1、VF2、VF3)时所获得的 2024 铝合金 Hill1948 本构参数表征结果。为验证两 方向、多虚场条件下各向异性屈服参数表征结果的 稳定性, 表征时采用了两组任意选取的不同初始值。 从表 2 可以看出, 由不同组初始值所获得的表征结
果基本相同，部分参数结果间细微的差别则是由试 验测量过程中不可避免的测量噪声等系统误差所引 起。表明当前试验构型下两方向、多虚场组合条件 可在不同初始猜测值下获得稳定的参数表征结果, 与文献 ${ }^{[24]}$ 结论一致。图 6 所示为目标函数收敛时(当 残差小于 $10^{-6}$ ), 两方向、三虚场所对应的内、外虚 功曲线。由图 6 可见, 各条内虚功曲线与对应外虚 功曲线一致性均较好, 验证了该条件下参数表征结 果的准确性。图 7 所示目标函数残差的变化过程则 证明了优化过程的可靠性。 
表 22024 铝合金 Hill1948 各向异性屈服参数表征结果

\begin{tabular}{|c|c|c|c|c|c|c|c|c|}
\hline 加载方向 & 虚场 & & $K / \mathrm{MPa}$ & $\varepsilon_{0}$ & $n$ & $H$ & $F$ & $N$ \\
\hline \multirow{4}{*}{$0^{\circ}+90^{\circ}$} & \multirow{4}{*}{$\begin{array}{l}\text { VF1 } \\
\text { VF2 } \\
\text { VF3 }\end{array}$} & 初始值 1 & 500 & 0.005 & 0.25 & 0.5 & 0.5 & 2.5 \\
\hline & & 结果 1 & 706 & 0.02 & 0.22 & 0.39 & 0.43 & 0.81 \\
\hline & & 初始值 2 & 1500 & 0.015 & 0.75 & 1.5 & 1.5 & 10 \\
\hline & & 结果 2 & 732 & 0.02 & 0.22 & 0.38 & 0.46 & 0.80 \\
\hline
\end{tabular}

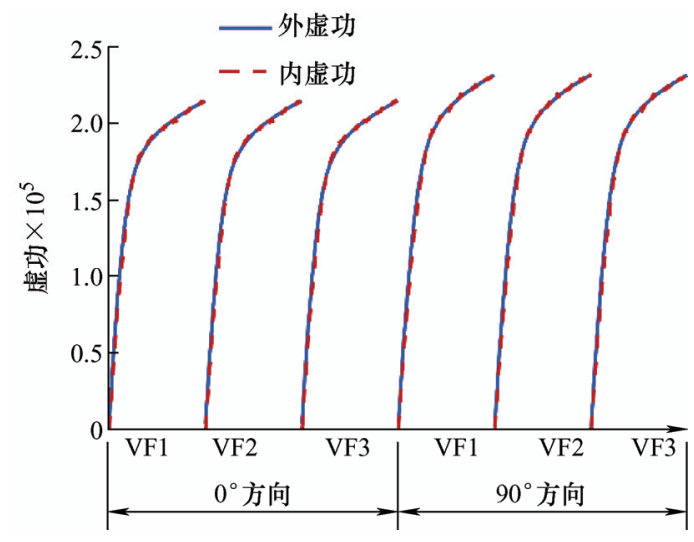

图 6 Hill1948 模型下目标函数收敛时两方向、三虚场 对应的内、外虚功曲线

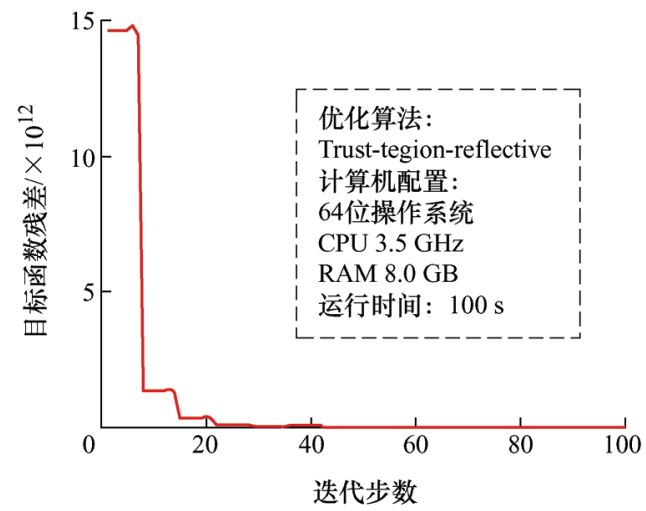

图 7 Hill1948 模型下目标函数残差的变化过程

为进一步验证所获得各向异性屈服参数的准确 性, 本文基于参数表征结果(表 2 中第一组结果)利 用 $A B A Q U S^{\circledR}$ 开展了相同构型和加载条件(同试件第 一个拉伸阶段, 包括 $0^{\circ}$ 和 $90^{\circ}$ 加载方向两个模型) 的有限元数值模拟。图 8 所示为桥型试件的有限元 模型, 采用壳单元 S3 划分网格。将仿真结果与试验 结果加以对比, 不难看出, 无论是试件的力-位移曲 线(图 9)还是对数应变分布云图(图 10), 仿真结果均 与试验测量结果具有较好一致性, 证明了该参数表 征结果是准确可靠的。此外, 如图 7 所示, 使用常 规配置的计算机，本方法的参数表征过程运算时间 仅需 $100 \mathrm{~s}$, 显示其高效性。相较于本文所使用的最 小二乘优化算法, 对于复杂非线性问题, 采用遗传
算法、模拟退火法 ${ }^{[25]}$ 、人工神经网络 ${ }^{[26]}$ 等优化算法则 有可能进一步提高计算精度和效率，缩短运算时间。

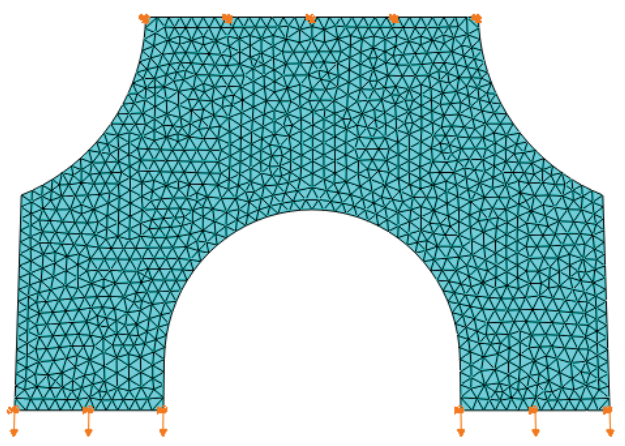

图 8 基于 Hill1948 的桥型试件单向拉伸有限元模型

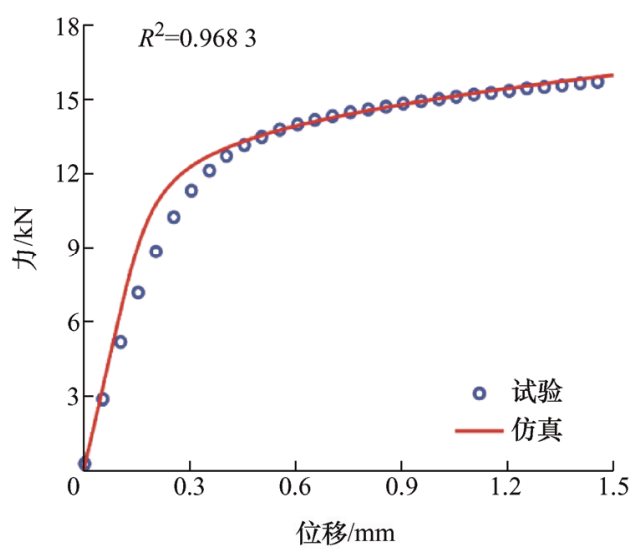

(a) $0^{\circ}$ 方向加载

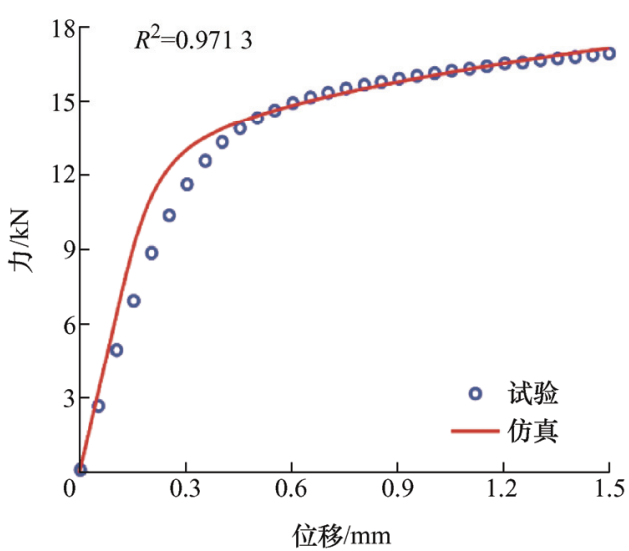

(b) $90^{\circ}$ 方向加载

图 9 基于 Hill1948 参数表征结果的仿真力-位移曲线 与对应的试验测量曲线 


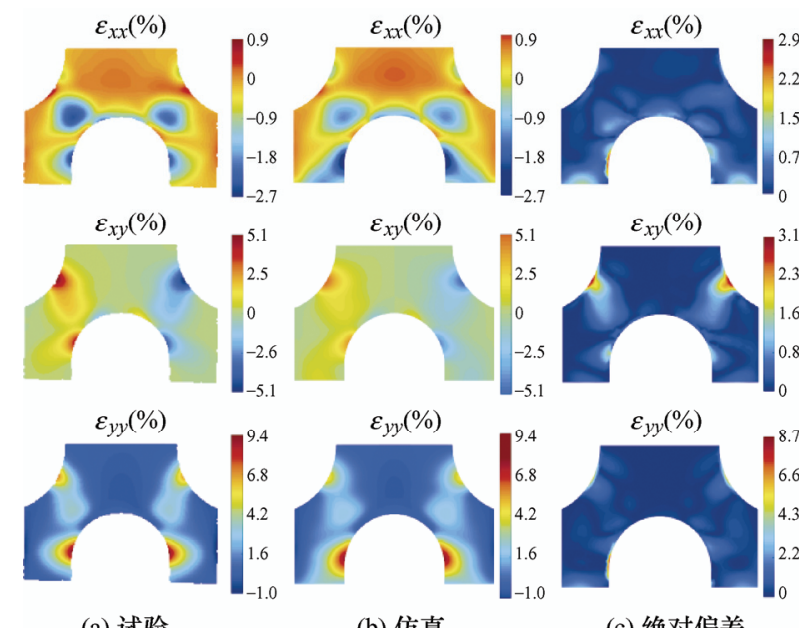

(a) 试验 (c) 绝对偏差

图 10 最后一个拉伸加载步下 $0^{\circ}$ 试件的试验测量应变云图 与对应的仿真应变云图

\section{2 非线性运动硬化模型参数表征结果}

基于单虚场(虚场 1), 利用试件屈曲前的两个拉

伸压缩循环试验数据分别对 $0^{\circ}$ 和 $90^{\circ}$ 方向加载的 试件进行了非线性运动硬化模型参数表征。由于选 取不同数量的背应力张量可对材料力学行为拟合效 果产生不同影响，本文同时考虑了单背应力张量和 双背应力张量两种情况。表 $3 、$ 表 4 所示为 $0^{\circ}$ 方向 加载时两种情况的参数表征结果。可以看出, 对于 不同初始值, 两种情况的收玫性均良好。图 11a 所 示为目标函数收敛时对应的内、外虚功曲线。结果 显示, 内、外虚功曲线整体一致性较好, 但在部分 区域存在少量偏差, 分析原因是由本构模型自身适 用性的局限所致。通过对比还可以看出, 双背应力 张量的内、外虚功曲线拟合程度与单背应力张量的 内、外虚功曲线拟合程度无明显差异, 图 12a 中两 种情况下目标函数残差的大小对比可验证这一点。 表明对于该材料使用单背应力张量即可满足材料非 线性运动硬化行为的有效预测。

表 3 非线性运动硬化模型参数表征结果 $\left(0^{\circ}\right.$ 方向, 单背应力张量 $)$

\begin{tabular}{|c|c|c|c|c|c|c|c|}
\hline 加载方向 & 虚场 & & $C / \mathrm{MPa}$ & $\gamma$ & $Y_{0} / \mathrm{MPa}$ & $R_{\text {sat }} / \mathrm{MPa}$ & $m$ \\
\hline \multirow{4}{*}{$0^{\circ}$} & \multirow{4}{*}{ VF1 } & 初始值 1 & 5000 & 100 & 100 & 30 & 30 \\
\hline & & 结果 1 & 5325 & 68.4 & 264 & $10^{-5}$ & $10^{-5}$ \\
\hline & & 初始值 2 & 15000 & 200 & 300 & 20 & 20 \\
\hline & & 结果 2 & 5329 & 68.4 & 264 & $10^{-5}$ & $10^{-5}$ \\
\hline
\end{tabular}

表 4 非线性运动硬化模型参数表征结果 $\left(0^{\circ}\right.$ 方向, 双背应力张量 $)$

\begin{tabular}{|c|c|c|c|c|c|c|c|c|c|}
\hline 加载方向 & 虚场 & & $C_{1} / \mathrm{MPa}$ & $\gamma_{1}$ & $C_{2} / \mathrm{MPa}$ & $\gamma_{2}$ & $Y_{0} / \mathrm{MPa}$ & $R_{\text {sat }} / \mathrm{MPa}$ & $m$ \\
\hline \multirow{4}{*}{$0^{\circ}$} & \multirow{4}{*}{ VF1 } & 初始值 1 & 10000 & 200 & 1000 & 50 & 100 & 50 & 50 \\
\hline & & 结果 1 & 14815 & 289 & 709 & $10^{-5}$ & 248 & $10^{-5}$ & $10^{-5}$ \\
\hline & & 初始值 2 & 30000 & 500 & 2000 & 30 & 300 & 30 & 30 \\
\hline & & 结果 2 & 14855 & 290 & 709 & $10^{-5}$ & 248 & $10^{-5}$ & $10^{-5}$ \\
\hline
\end{tabular}

对于 $90^{\circ}$ 方向加载的试件，表 5 和表 6 给出了 两种背应力张量下的参数表征结果。与 $0^{\circ}$ 加载时相 同, 不同初始值均收玫于一致的结果。从图 $11 \mathrm{~b}$ 和 图 $12 \mathrm{~b}$ 可以看出, $90^{\circ}$ 方向加载时内、外虚功曲线同 样具有一致性, 且两种背应力张量的内、外虚功曲线 拟合效果亦无明显差异, 这与先前 $0^{\circ}$ 方向试件的结
论一致。值得注意的是, 表 $3 \sim 6$ 中几种情况下 $R_{\text {sat }}$ 和 $m$ 均趋于零(收玫于下限值 $10^{-5}$ ), 即当前流动应力 $\sigma_{\mathrm{s}}$ 趋近于常数 $Y_{0}\left(\right.$ 因 $R_{\mathrm{sat}}\left(1-\exp \left(-m \varepsilon_{\mathrm{p}}\right)\right) \approx 0$ ，表明对 于本研究中的 2024 铝合金, 采用当前的非线性运动 硬化模型, 在金属塑性变形过程中, 材料的屈服面仅 发生了移动而未发生扩大。

表 5 非线性运动硬化模型参数表征结果 $\left(90^{\circ}\right.$ 方向, 单背应力张量)

\begin{tabular}{|c|c|c|c|c|c|c|c|}
\hline 加载方向 & 虚场 & & $C / \mathrm{MPa}$ & $\gamma$ & $Y_{0} / \mathrm{MPa}$ & $R_{\text {sat }} / \mathrm{MPa}$ & $m$ \\
\hline \multirow{4}{*}{$90^{\circ}$} & \multirow{4}{*}{ VF1 } & 初始值 1 & 5000 & 100 & 100 & 30 & 30 \\
\hline & & 结果 1 & 8663 & 37.3 & 252 & $10^{-5}$ & $10^{-5}$ \\
\hline & & 初始值 2 & 15000 & 500 & 500 & 20 & 20 \\
\hline & & 结果 2 & 8663 & 37.2 & 252 & $10^{-5}$ & $10^{-5}$ \\
\hline
\end{tabular}


表 6 非线性运动硬化模型参数表征结果 $\left(90^{\circ}\right.$ 方向, 双背应力张量)

\begin{tabular}{|c|c|c|c|c|c|c|c|c|c|}
\hline 加载方向 & 虚场 & & $C_{1} / \mathrm{MPa}$ & $\gamma_{1}$ & $C_{2} / \mathrm{MPa}$ & $\gamma_{2}$ & $Y_{0} / \mathrm{MPa}$ & $R_{\text {sat }} / \mathrm{MPa}$ & $m$ \\
\hline \multirow{4}{*}{$90^{\circ}$} & \multirow{4}{*}{ VF1 } & 初始值 1 & 10000 & 200 & 1000 & 50 & 100 & 50 & 50 \\
\hline & & 结果 1 & 13611 & 141 & 1992 & $10^{-5}$ & 236 & $10^{-5}$ & $10^{-5}$ \\
\hline & & 初始值 2 & 30000 & 500 & 2000 & 30 & 300 & 30 & 30 \\
\hline & & 结果 2 & 13655 & 141 & 1995 & $10^{-5}$ & 236 & $10^{-5}$ & $10^{-5}$ \\
\hline
\end{tabular}

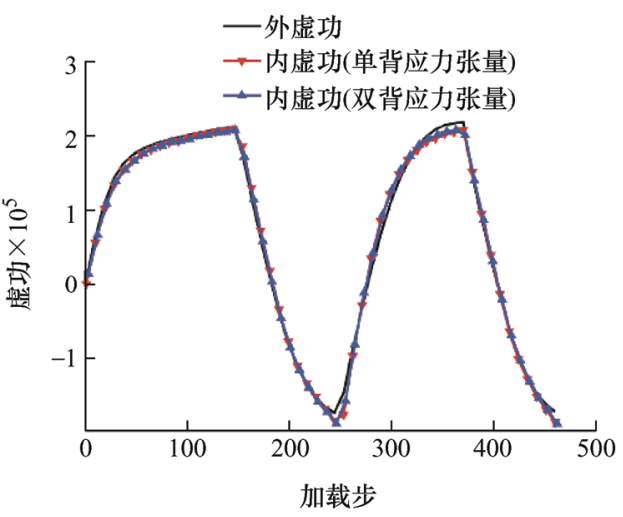

(a) $0^{\circ}$ 方向加载

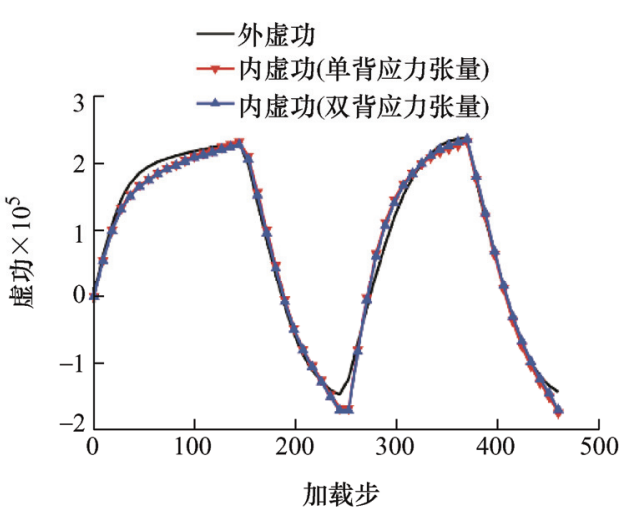

(b) $90^{\circ}$ 方向加载

图 11 非线性运动硬化模型下不同加载方向和背应力张量 条件对应的内、外虚功曲线

同先前的数值模拟验证，基于表 4 和表 6 中第 一组表征结果分别开展了相同条件的桥型试件循环 拉伸-压缩有限元仿真 $\left(0^{\circ}\right.$ 和 $90^{\circ}$ 方向加载, 双背应 力张量)。图 13 所示为两种情况下的试验与仿真力位移曲线。由图 13 可见，除部分区域存在一定差异 外, 二者整体表现出一致性, 该运动硬化模型能够 有效捕捉 2024 铝合金反向加载时的包辛格效应。 图 14 为 $0^{\circ}$ 试件屈曲前最后一个压缩加载步时, 数 字图像相关测量得到的对数应变云图与对应的仿真 应变云图, 二者同样具有近似的分布情况。需要指 出的是, 在试件底部等局部区域, 试验应变的分布 形式与仿真应变存在一定差异(图 10 中亦可观察到 此现象)。分析其原因, 是由于试验中试验机夹头产

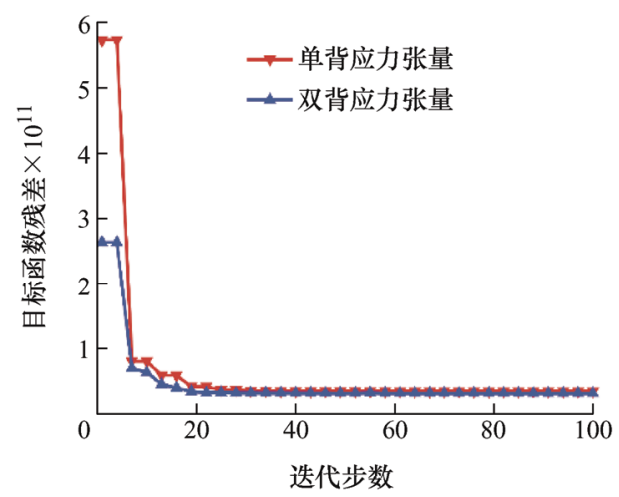

(a) $0^{\circ}$ 方向加载

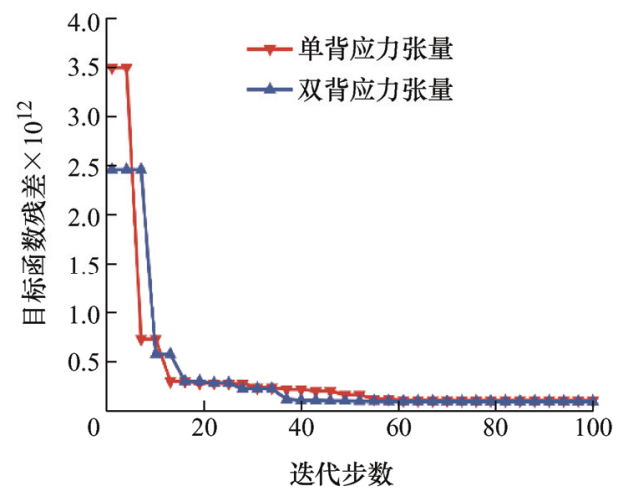

(b) $90^{\circ}$ 方向加载

图 12 不同加载方向和背应力张量条件下目标函数残差的 变化过程

生的试件边界条件与有限元仿真中所定义的模型边 界条件存在差异, 试验机夹头对试件边界存在一定 的夹紧力和摩擦力作用, 而后者则无此作用, 这种 差异可在一定程度上影响试件局部的应变分布, 特 别是应变集中区域的发生位置，导致仿真与试验结 果在局部对应点产生较明显的偏差。以上结果验证 了 Hill1948 屈服准则和非线性运动硬化模型对 2024 铝合金各向异性塑性力学行为预测的适用性，本文 所提出的表征方法亦可应用于其他先进各向异性塑 性模型, 例如结合 YLD2000-2D 屈服准则 ${ }^{[6]}$ 和 HAH、 $\mathrm{HAH} 20$ 等模型 ${ }^{[10,27]}$, 通过采用高阶多参数屈服函数 和扭曲旋转型屈服面, 有望进一步提高材料各向异 性塑性力学行为预测的准确性。 


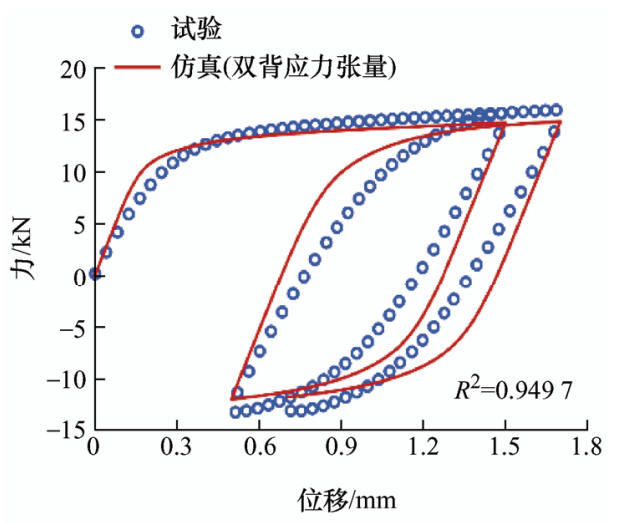

(a) $0^{\circ}$ 方向加载

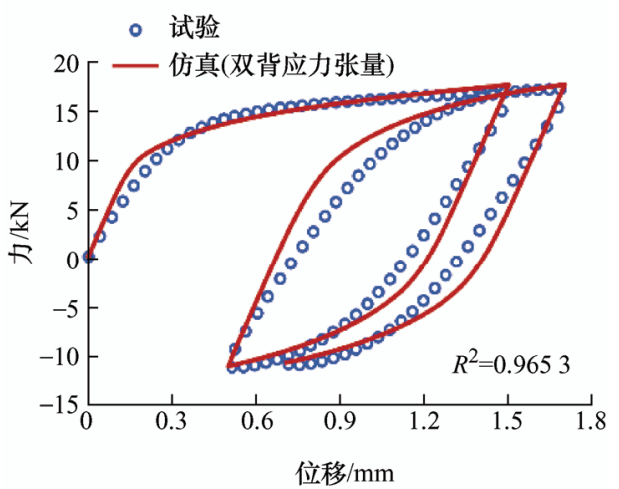

(b) $90^{\circ}$ 方向加载

图 13 基于非线性运动硬化模型参数表征结果的 仿真力-位移曲线与对应的试验测量曲线

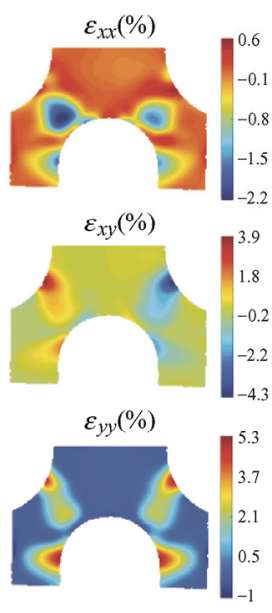

(a) 试验

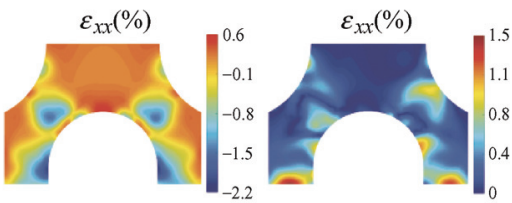

$\varepsilon_{x y}(\%)$

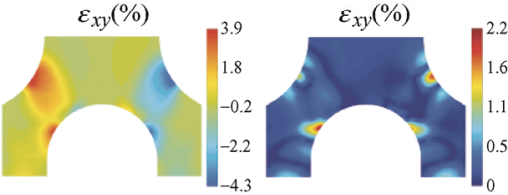

$\varepsilon_{y y}(\%)$

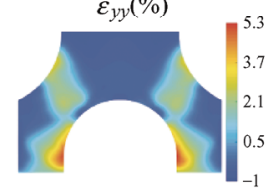

(b) 仿真

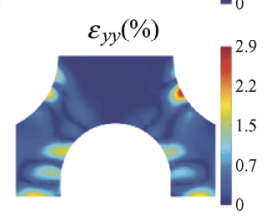

(c) 绝对偏差
图 14 最后一个压缩加载步下 $0^{\circ}$ 试件的试验测量应变云图 与对应的仿真应变云图

\section{4 结论}

(1) 针对 2024 铝合金轧制板材, 提出了基于桥 型试件循环拉伸-压缩试验、数字图像相关全场变形 测量和虚场反问题求解法, 开展材料各向异性塑性 属性表征研究。该研究方法可在常规单轴万能试验
机上, 通过最少数量的力学试验, 实现材料各向异 性屈服与塑性硬化多参数的高效和同步表征，大幅 减少试验数量, 简化试验过程。

（2）针对 Hill1948 各向异性屈服模型, 在参数 优化目标函数中结合材料 $0^{\circ}$ 和 $90^{\circ}$ 两拉伸加载 方向试验数据, 同时采用多虚场约束, 可在不同 初始猜测值条件下获得稳定可靠的参数表征结 果。基于所表征参数而开展的有限元数值模拟与 试验测量结果具有良好的一致性, 有效验证了参 数表征的准确性。

(3) 对于非线性运动硬化模型, 采用单材料方 向试验数据和单虚场的目标函数即可获得对应材料 方向稳定可靠的非线性运动硬化参数表征结果。内、 外虚功曲线对比表明, 当前运动硬化模型对 2024 板材 $0^{\circ}$ 和 $90^{\circ}$ 材料方向的力学行为表征适用性无 明显差异。此外, 采用当前的非线性运动硬化模型, 材料塑性变形过程中屈服面仅发生了移动而未发生 扩大。

\section{参 考 文 献}

[1] 华家辉, 徐从昌, 林天豪, 等. 6 XXX 铝合金防撞梁总 成在动静态载荷下的变形行为 [J]. 塑性工程学报, 2019，26(6): 199-205.

HUA Jiahui, XU Congchang, LIN Tianhao, et al. Deformation behavior of 6XXX aluminum alloy anti-collision beam assembly under dynamic and static loading[J]. Journal of Plasticity Engineering, 2019, 26(6): 199-205.

[2] 杨合, 詹梅, 李甜, 等. 铝合金大型复杂薄壁壳体旋压 研究进展 [J]. 中国有色金属学报, 2011，21(10): 2534-2550.

YANG He, ZHAN Mei, LI Tian, et al. Advances in spinning of aluminum alloy large-sized complicated thin-walled shells $[\mathrm{J}]$. Chinese Journal of Nonferrous Metals, 2011, 21(10): 2534-2550.

[3] ZANCHETTA B D, SILVA V, SORDI V L, et al. Effect of asymmetric rolling under high friction coefficient on recrystallization texture and plastic anisotropy of AA1050 alloy[J]. Transactions of Nonferrous Metals Society of China, 2019, 29(11): 2262-2272.

[4] 李小强, 董红瑞, 于长旺, 等. 不同屈服准则与硬化模 型对 DP780 双相高强钢拉延弯曲回弹预测影响规律研 究[J]. 机械工程学报, 2020, 56(12): 42-55.

LI Xiaoqiang, DONG Hongrui, YU Changwang, et al. Influence of yield criteria and hardening model on 
draw-bending spring back prediction of DP780[J]. Journal of Mechanical Engineering，2020，56(12): 42-55.

[5] HILL R. A theory of the yielding and plastic flow of anisotropic metals $[\mathrm{J}]$. Proceedings of the Royal Society of London, 1948, 193(1033): 281-297.

[6] BARLAT F, BREM J C, YOON J W, et al. Plane stress yield function for aluminum alloy sheets-Part 1 : theory[J]. International Journal of Plasticity, 2003, 19(9): 1297-1319.

[7] FREDERICK C O, ARMSTRONG P J. A mathematical representation of the multiaxial Bauschinger effect[J]. Materials at High Temperatures, 2007, 24(1): 1-26.

[8] CHABOCHE J L. Time-independent constitutive theories for cyclic plasticity[J]. International Journal of Plasticity, 1986, 2(2): 149-188.

[9] YOSHIDA F, UEMORI T. A model of large-strain cyclic plasticity describing the Bauschinger effect and workhardening stagnation[J]. International Journal of Plasticity, 2002, 18(5-6): 661-686.

[10] BARLAT F, GRACIO J J, LEE M G, et al. An alternative to kinematic hardening in classical plasticity[J]. International Journal of Plasticity, 2011, 27(9) : 1309-1327.

[11] 王海波, 门明良, 阎昱, 等. 比例/非比例加载路径下 57540 汽车用铝合金板各向异性变形行为的精确解析 [J]. 机械 工程学报, 2018, 54(24): 91-101.

WANG Haibo, MEN Mingliang, YAN Yu, et al. Accurate analysis of anisotropic deformation behavior of 57540 aluminum alloy sheet under proportional/non-proportional loading paths[J]. Journal of Mechanical Engineering, 2018, 54(24): 91-101.

[12] DICK R, YOON J W. Plastic anisotropy and failure in thin metal: Material characterization and fracture prediction with an advanced constitutive model and polar EPS (effective plastic strain) fracture diagram for AA 3014-H19[J]. International Journal of Solids and Structures, 2018, 151: 195-213.

[13] CHOI J H, ZANG S L, LEE M G, et al. Determining the coefficients of a homogeneous anisotropic hardening model for ultrathin steel sheets[J]. International Journal of Mechanical Sciences, 2019, 157-158: 428-438.

[14] LEE J, KIM S J, LEE Y S, et al. Distortional hardening concept for modeling anisotropic/asymmetric plastic behavior of AZ31B magnesium alloy sheets[J]. International Journal of Plasticity，2017，94: 74-97.

[15] 李群, 金永, 邹宗园, 等. 基于循环拉-压试验的混合
硬化模型参数确定及模型应用 $[\mathrm{J}]$. 机械工程学报, 2020, 56(2): 63-68.

LI Qun, JIN Miao, ZOU Zongyuan, et al. Parameter determination and application research of mixed hardening model based on cyclic tension-compression test[J]. Journal of Mechanical Engineering, 2020，56(2): 63-68.

[16] CHOI J S, LEE J, BARLAT F, et al. Measurement and modeling of simple shear deformation under load reversal: Application to advanced high strength steels $[\mathrm{J}]$. International Journal of Mechanical Sciences，2015，98: 144-156.

[17] SCHREIER H, ORTEU, SUTTON M A, et al. Image correlation for shape, motion and deformation measurements: Basic concepts, theory and applications[M]. Boston: Springer, 2009.

[18] ZHANG S, LEOTOING L, GUINES D, et al. Calibration of anisotropic yield criterion with conventional tests or biaxial test[J]. International Journal of Mechanical Sciences, 2014, 85: 142-151.

[19] PIERRON F, GREDIAC M. The virtual fields method: Extracting constitutive mechanical parameters from full-field deformation measurements[M]. New York : Springer, 2012.

[20] FU J, BARLAT F, KIM J H, et al. Application of the virtual fields method to the identification of the homogeneous anisotropic hardening parameters for advanced high strength steels[J]. International Journal of Plasticity, 2017, 93: 229-250.

[21] MARTINS J M P, ANDRADE-CAMPOS A, THUILLIER S. Calibration of anisotropic plasticity models using a biaxial test and the virtual fields method[J]. International Journal of Solids and Structures, 2019, 172-173: 21-37.

[22] FU J, XIE W, QI L. An identification method for anisotropic plastic constitutive parameters of sheet metals[J]. Procedia Manufacturing, 2020, 47: 812-815.

[23] SUTTON M A, DENG X, LIU J, et al. Determination of elastic-plastic stresses and strains from measured surface strain data[J]. Experimental Mechanics, 1996, 36(2): 99-112.

[24] ROSSI M, PIERRON F, ŠTAMBORSKÁ M. Application of the virtual fields method to large strain anisotropic plasticity[J]. International Journal of Solids and Structures, 2016, 97-98: 322-335.

[25] 王雪梅, 王义和. 模拟退火算法与遗传算法的结合 $[\mathrm{J}]$. (下转第 88 页) 
纳米压痕变形行为研究 [J]. 机械工程学报, 2018, 54(8):

151-156.

XU Lianyong, ZHANG Shuting, JING Hongyang, et al. Research on the deformation behavior of Ag-GNSs/ $\mathrm{SnAgCu}$ solders during nanoindentation tests[J]. Journal of Mechanical Engineering, 2018，54(8): 151-156.

[20] JOHNSON K L. Contact mechanics[M]. Cambridge: Cambridge University Press, 1985.

[21] HYUN H C, KIM M, LEE J H, et al. A dual conical indentation technique based on FEA solutions for property evaluation[J]. Mechanics of Materials, 2011, 43(6): 313-331.

[22] 张赜文, 余家欣, 钱林茂. 压头曲率半径对单晶硅径向
纳动损伤的影响 $[\mathrm{J}]$. 机械工程学报, 2010, 46(9): 107-112.

ZHANG Zewen, YU Jiaxin, QIAN Linmao. Effect of curvature radius of indenter on the radial nanofretting damage of monocrystal silicon[J]. Journal of Mechanical Engineering, 2010, 46(9): 107-112.

作者简介: 陈辉, 男, 1990 年出生, 院聘副教授。主要研究方向为材料 测试理论与方法。

E-mail: chen_hui5352@163.com

彭晖(通信作者), 男, 1976 年出生, 教授, 博士研究生导师。主要研究 方向为新材料与新型结构的力学与耐久性能。

E-mail: huipeng@esust.edu.cn

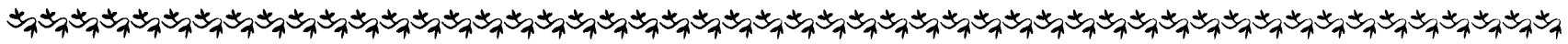

(上接第 78 页)

计算机学报, 1997, 20(4): 381-384.

WANG Xuemei, WANG Yihe. The combination of simulated annealing and genetic algorithms[J]. Chinese Journal of Computers, 1997, 20(4): 381-384.

[26] 杨源杰, 黄道. 人工神经网络算法研究及应用[J]. 华东 理工大学学报, 2002, 28(5): 115-118.

YANG Yuanjie, HUANG Dao. Research and applications of artificial neural networks[J]. Journal of East China University of Science and Technology, 2002, 28(5): 115-118.

[27] BARLAT F, YOON S Y, LEE S Y, et al. Distortional plasticity framework with application to advanced high strength steel[J]. International Journal of Solids and Structures, 2020，202: 947-962.

作者简介: 付佳伟, 男, 1983 年出生, 博士, 副教授, 硕士研究生导 师。主要研究方向为新型材料力学属性反问题求解理论、数值模拟和 测试技术。

E-mail: jiawei.fu@nwpu.edu.cn 齐乐华(通信作者), 女, 1957 年出生, 博士, 教授, 博士研究生导师。 主要研究方向为轻质合金基复合材料成形工艺及理论。

E-mail: qilehua@nwpu.edu.cn 